Article

\title{
Advancements in Passenger Processes at Airports from Aircraft Perspective
}

\author{
Michael Schultz ${ }^{1, * \mathbb{D}}$ and Michael Schmidt ${ }^{2}$ \\ 1 Institute of Flight Guidance, German Aerospace Center (DLR e.V.), 38108 Braunschweig, Germany \\ 2 Bauhaus Luftfahrt e.V., 82024 Taufkirchen, Germany; m.schmidt@munich-airport.de \\ * Correspondence: michael.schultz@dlr.de
}

Received: 21 September 2018; Accepted: 22 October 2018; Published: 25 October 2018

\begin{abstract}
We provide an overview about the research done in the field of airport and airline operations with a specific focus on a fast, reliable and sustainable passenger boarding. The reliable prediction operational processes along the aircraft air-ground trajectory demands a comprehensive consideration of economic, environmental, and handling constraints of airlines and airports. In particular, the critical process of passenger boarding is driven by passengers' ability to follow the proposed boarding procedures and is not controlled by operational experts. In this paper we implement and compare two individual-based approaches which cover both specific passenger behavior during boarding and operational airline constraints. Both models used similar input values, but exhibit different magnitudes in the benefit evaluation. Furthermore, we demonstrate that there are still unused potentials to further improve boarding progress by using innovative infrastructural adaptations inside the aircraft cabin.
\end{abstract}

Keywords: passenger boarding; infrastructural adaptations; Side-Slip Seat; cinema seat; aircraft turnaround; model comparison

\section{Introduction}

The proposed annual 4.4-4.7\% growth in passenger traffic in the next 20 years [1,2] will challenge the aviation industry significantly. It is imperative that advancements in process sustainability and reliability as well as passenger comfort are developed to deal with increasing number of travelers at major hub airports to reach the ambitious Flightpath 2050 targets, such as $90 \%$ of travelers within Europe are able to complete their journey, door-to-door within $4 \mathrm{~h} \mathrm{[3].} \mathrm{The} \mathrm{aircraft} \mathrm{turnaround} \mathrm{at}$ airports, as important part of the aircraft trajectory over the day of operations, has to be part of sustainable optimization strategies for minimizing flight delays [4] and ensuring flight connection considering operational uncertainties (cf. [5,6]). In this context, turnaround absorbs inbound delay [7] and could enhance slot adherence at airports [8] or mitigate problems of push-back scheduling [9]. Current research in the field of air traffic management primarily addresses the economic and ecological impact of flight trajectories (cf. [10-13]) but has to include efficient aircraft ground operations as well in order to ensure an efficient aircraft trajectory over the day of operations [14]. In this context, research was conducted with regards to the turnaround performance implementing collaborative management [15] or addressing the future airport performance [16].

The trend towards denser aircraft cabins driven by both low cost carrier and larger leads to an increase of the average number of installed seats per aircraft on short-haul segments from 110 to 160 since 1960 [17]. Accordingly, the passenger boarding become more and more apparent as a critical process during aircraft ground operations [18]. It is the last step of various landside handling processes (passenger trajectory), such as check-in and security check, which passenger have to follow in the airport terminal $[19,20]$. From the airside perspective, the aircraft trajectory at the day of 
operations depends on efficient arrival/departure procedures and en-route performance, but also on a reliable and efficient aircraft turnaround at the airport. The turnaround consists of the major tasks disembark, cater, clean, fuel, boarding (and parallel loading/unloading), which are controlled by ground handling agents. In this context, the boarding process is driven by passengers experience and ability to follow the proposed procedures (e.g., late arrivals, no shows, amount of hand luggage, status passengers, cf. [21-23]). Different airline services (e.g., priority boarding), an increased amount of hand luggage as well as changing passenger demographics (composition, behavior, anthropometrics) results in a decrease of average boarding speed from around 20 passengers per minute to nearly nine passengers per minute. Furthermore, the cabin layout, aircraft configuration, and the operational airport environment (e.g., gate or apron positions) influence the passenger boarding process.

The paper will focus on the evaluation of alternative boarding strategies to reliably increase the passenger boarding efficiency even under operational constraints. Various boarding strategies have been applied by airlines, which introduce a predefined sequence of passengers entering the aircraft cabin depending on their seats. However, most of the strategies tested are not sustainable for regular or future flight operations. Novel approaches aim to reduce operational drawbacks and call for a parallelization of the boarding processes which showed a more efficient use of the aisle [24-27]. Since the stability and predictability of the boarding progress have to be focused to provide sustainable approaches for the aircraft boarding, we address innovative seat concepts with significant impact to current cabin infrastructure and passenger boarding time.

\subsection{Status Quo}

In the following section, a short overview concerning scientific research on aircraft boarding is given. Relevant studies concerning aircraft boarding strategies include but are not limited to the following examples. More comprehensive overviews are provided by Jaehn and Neumann and Schultz for the passenger boarding [28,29] and by Schmidt for the aircraft turnaround [30]. A common goal of simulation-based evaluations is to minimize the time that is required for passenger boarding. Van Landeghem and Beuselinck [31] investigates the potential of optimized boarding strategies considering different boarding patterns. A similar approach is used by Ferrari and Nagel [32], particularly focused on disturbances on the boarding sequence caused by early or late arrivals of passengers. The results show improved values for the typical back-to-front boarding in the case of passengers not boarding in their previously assigned boarding groups. In contrast, Bachmat and Elkin [33] support the back-to-front policy in comparison to random boarding strategy. The interference of passengers when boarding an aircraft is in the focus of a study by Bazargan [34]. A stochastic approach to cover both individual passenger behavior and aircraft/airline operational constraints (Schultz et al. [21]) exhibits that the efficiency of the back-to-front policy depends on the size of the boarding blocks (seat rows are aggregated to a boarding block).

On the basis of an individual boarding strategy proposed by Steffen [24], which considers the time a passenger needs to store baggage, the model developed by Milne and Kelly [25] assigns passengers to seats so that their hand luggage is distributed evenly throughout the plane. Since individually boarding strategies often split passenger travelling together, Zeineddine [27] proposed a dynamically optimized boarding strategy where passengers are sequenced in a boarding queue based on their seats' positions, associated groups, and the possibility of interferences, immediately after the last check-in. Chung [35] addresses the aircraft seating layout and indicates that alternative designs could significantly reduce the boarding time. A link between the efficiency of an airline's boarding policies and the aircraft design parameters, such as distance between the rows, is given in a study by Bachmat et al. [36]. In this study, results show a higher attractiveness of random boarding among row-based policies.

Focusing on the simulation of different deplaning strategies, several types are tested in a study by Wald et al. [37]. Picking up the idea of boarding groups, a study based on an analytical model by van den Briel et al. [38] shows a significantly improved boarding time by group boarding policies over the back-to-front policy. Based on a mathematical model that is related to the $1+1$ polynuclear 
growth model with concave boundary conditions, Bachmat et al. [39] study all aircraft configurations and boarding group sizes. Results show that the effectiveness of back-to-front boarding can be increased compared to random boarding but drops when having more than two boarding groups. Assessing the effectiveness of boarding strategies is also a core part of a study by Soolaki et al. [40]. Based on an integer linear programming approach together with a genetic algorithm, they analyze different boarding strategies to assess the effectiveness of their model. The mathematical model's output aims to minimize the interferences and to speed up the boarding time as interferences may lead to delays, especially in single-aisle aircraft. The interactions of passengers during the boarding process (e.g., occupied aisle) are also in the focus of the studies provided by Frette and Hemmer [41], Tang et al. [42], and Schultz et al. [22]. Frette and Hemmer calculate the average boarding time with a dynamical model, assuming that all permutations of the amount of passengers have the same weight. Tang et al. concentrate on the passenger's individual properties and apply this knowledge to their numerical model in order to evaluate the benefit of different boarding strategies. Schultz extends the stochastic boarding model to analyze twin-aisle configurations. Fuchte [17] focusses on the aircraft design and, in particular, the impact of aircraft cabin modifications with regard to the boarding efficiency. Schmidt et al. [43] and Schmidt and Heinemann [44] evaluate novel aircraft layout configurations and seating concepts for regional, single- and twin-aisle aircraft with 50-300 seats. Schultz [45] implemented the innovative Side-Slip Seat [46] and demonstrates benefit during passenger boarding, when passengers can pass each other in the aircraft aisle.

In the context of calibration (input parameter) and validation (simulation results), only few experimental tests have been conducted by the research community. Steffen and Hotchkiss [47] tested airplane boarding methods in a mock Boeing 757 fuselage. Kierzkowski and Kisiel [48] provide an analysis covering the time needed to place items in the overhead bins depending on the availability of seats and occupancy of the aircraft. Miura and Nishinari [49] conducted an experiment to understand how passengers assessed boarding/deboarding times. Schultz [50] provides a comprehensive set of operational field trial data including classification of boarding times, passenger arrival times, time to store hand luggage, and passenger interactions in the aircraft cabin as a fundamental basis for boarding model calibration. Gwynne et al. [51] perform a series of small-scale laboratory tests to help quantify individual passenger boarding and deplaning movement considering seat pitch, hand luggage items, and instructions for passengers.

\subsection{Document Structure}

In this paper we provide a comparison of two approaches to cover the individual behavior of passengers during aircraft boarding. After a brief introduction of the different model approaches, we investigate the general impact of the number of hand luggage pieces to the boarding time and their distribution in boarding sequence (e.g., passengers with high number of bags board first). The implementation of two innovative infrastructural adaptations (Side-Slip Seat and cinema seat) demonstrates unused potentials to sustainably improve the passenger boarding process. The last evaluation indicates how groups of passengers will influence the boarding process. Finally, a brief discussion emphasizes the need for adapted procedures when the infrastructure is changed and addresses the transferability of evaluation results (scaling effects). In the outlook, we show how a connected aircraft cabin could be used for a dynamic seat allocation to sustainably improve future passenger boarding.

\section{Boarding Models}

Two individual-based models are used for the comparative analysis of different boarding strategies: paxSim and PAXelerate. Both models are based on a microscopic, individual approach to cover individual passenger behaviors and operational constraints. The following sections provide a brief overview about the two models. 


\subsection{Stochastic Approach-paxSim}

The passenger boarding is assumed as a stochastic transition and modeled as an asymmetric simple exclusion process (ASEP, cf. Schultz [52]). Herein, passenger movements are assumed to be an one-dimensional, forward-directed, and discrete (time and space) process. This ASEP approach was already successfully implemented to cover passenger behavior in airport terminals $[19,20]$. The common aircraft seat layout consists of regular structures, which could be appropriately transferred into a grid with aircraft entries, aisle(s) and passenger seats as shown in Figure 1 (reference: Airbus 320, 29 rows, 174 seats). This regular grid is implemented in a cell-based simulation environment, whereas cells have a size of $0.4 \times 0.4 \mathrm{~m}$ and can either be empty or contain exactly one passenger.
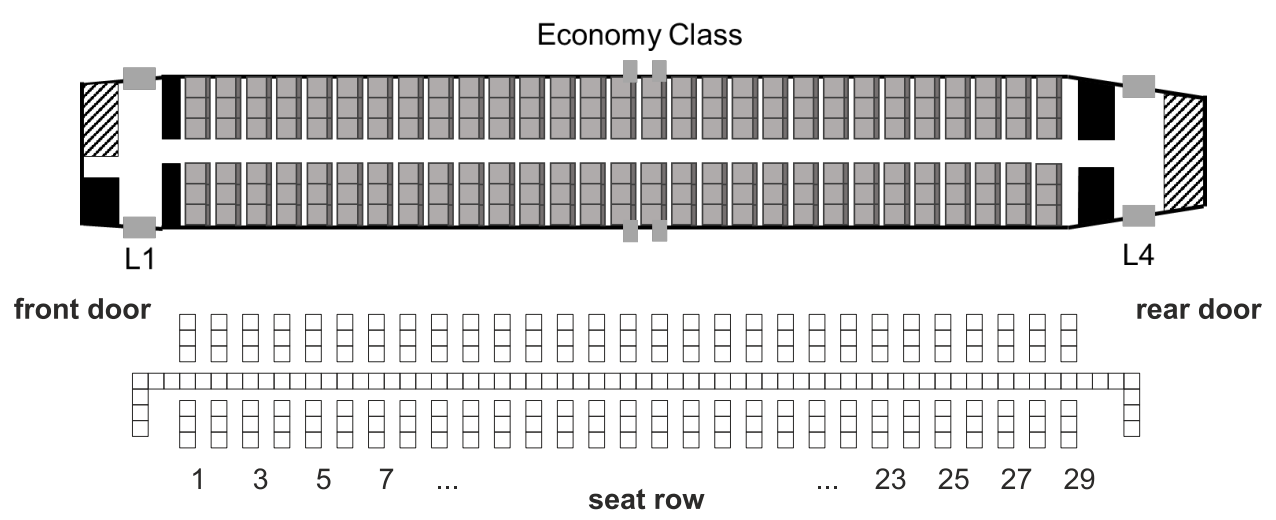

Figure 1. (a) A320 reference cabin layout (single aisle with front door at L1 and rear door at L4); (b) corresponding regular grid structure of the A320 model in the simulation environment.

A simple set of rules are defined to model the individual passenger movement: (a) enter the aircraft at the assigned door (based on the current boarding scenario), (b) move forward from cell to cell along the aisle until reaching the assigned seat row, and (c) store the hand luggage (aisle is blocked for other passengers) and take the seat. Thus, passenger movements depend on individual parameters (e.g., assigned seat, walking speed) and on local interactions, defined by the state of the adjacent cell (empty or occupied). When the passenger arrives the assigned seat row, the hand luggage storage is also modeled as a stochastic process, which depends on the individual amount of hand luggage pieces and a probability distribution for storage time. Finally, the time to take the seat is also covered by probability distributions for the individual seating time and additionally depends on the conditions in corresponding row (already used seats).

In order to derive reliable simulation results, the developed stochastic approach of aircraft boarding requires a minimum of simulation runs for an evaluation of a specific boarding strategy. In the simulation environment, a evaluation scenario is defined by the aircraft seat layout, the passenger number (derived from a seat load factor, default: 85\%), the number of used aircraft doors (default 1 door), the arrival frequency of the passengers at the aircraft (default: 14 passengers per minute), the specific boarding strategy (default: random) and the passenger compliance according to this boarding strategy (default: $85 \%$ ). Further details regarding passenger model, simulation environment, and calibration/validation are provided by Schultz $[23,50]$.

\subsection{PAXelerate}

PAXelerate $[53,54]$ is a passenger flow simulation framework and based on a microscopic approach applying agent-based modeling techniques. Each passenger is represented by an agent with individual properties such as walking speed, body dimensions, target seat or type of hand luggage. This allows agent interactions to be modeled to capture the resulting complex system behavior. Agents are generated, taking into account their anthropometrics and assigned seats. The anthropometric properties of waist width, body depth and walking speed are determined using a normal distribution 
between minimum and maximum values. Based on the passenger's age, the appropriate walking speed is derived. Diverse behavior patterns mainly influence the simulation process in terms of agents' overtaking behavior. Furthermore, hand luggage impacts the individual walking speed and requires additional stowing time to be performed before seating.

PAXelerate enables dynamic reactions to be modeled based on the mood and environment and various passenger's patterns to be defined, such as business or leisure travelers. The boarding sequence can be set using predefined boarding strategies. Agents use an A-Star pathfinding algorithm to search for the shortest and most cost efficient path to their assigned seat. The simulation foundation is based on a grid enabling the agent to move in eight directions. Each node on the grid possesses properties such as location, neighbors and occupation status, as well as, distance and cost which are important during the pathfinding. A gradient-based potential is defined around the cabin monuments and agents permitting the agents to walk directly next to the obstacles. Thus, agents could follow the pre-calculated path and appropriately react on obstacles occurring on the way to the assigned seat. The agent is able to turn in 45-degree steps allowing for a step sideways.

\section{Operational and Procedural Improvements}

To improve the aircraft boarding process, several approaches are developed. In this section, we will focus on the hand luggage (number of items and distribution in the cabin), infrastructural changes (Side-Slip Seat [46], cinema seat [55]), and the impact of groups (two members and two rows with 6 members). To cover the impact to the passenger boarding, three different boarding strategies are used as a reference: random, block, and outside-in boarding. The random boarding is defined by no specific chronological order of the passengers arriving at boarding gate. For the block boarding, the aircraft is divided into six blocks, where each block contains of five seat rows (last block has four rows), and these blocks are boarded form the back of the aircraft to the front. The outside-in boarding is defined by three boarding groups: the first group contains all passengers seated on the window, followed by a group with all passengers seated on the middle seat, and the last group contains all aisle seated passengers. This boarding strategy is also named as WilMA (window, middle, aisle). In the evaluation, the different strategies are used as follows: random boarding as baseline, block boarding as commonly used strategy, and outside-in as reference for a fast boarding strategy.

\subsection{Number of Hand Luggage Items}

Two frequently statements with regard to the hand luggage items are: (a) if passengers have no hand luggage, the boarding will be immediately faster, and (b) a large amount of hand luggage leads to blocked overhead compartments and results in counterflow conditions in the aircraft aisle, when passengers are looking for empty compartments. In the prior analysis, the focus was more set on the boarding sequence optimization to prevent unfavorable seat row states [21,22], where the amount of hand luggage was taken as an external, unchanged parameter. Besides the expected positive effects on the boarding time, passengers could perceive a reduced amount of hand luggage items as a degrade level of service caused by additional baggage drop and pickup processes in the (congested) check-in and baggage claim areas in the airport terminal. These processes will results in additional waiting times for the individual passenger. In Figure 2 the passenger boarding time is shown in relation to the number of hand luggage items.

The application of both the stochastic paxSim and PAXelerate results in a decreasing boarding time, when the number of items is reduced. PAXelerate calculates are higher degree of dependency between the number of items and the boarding time: $-75 \%$ boarding time for random boarding in contrast to $-34 \%$ boarding time using paxSim. On the other hand, paxSim indicates a higher performance of the block boarding, if the number of items are smaller than 0.45 items per passenger at average. In a two-door configuration, the block and the random boarding demonstrate a comparable behavior. 

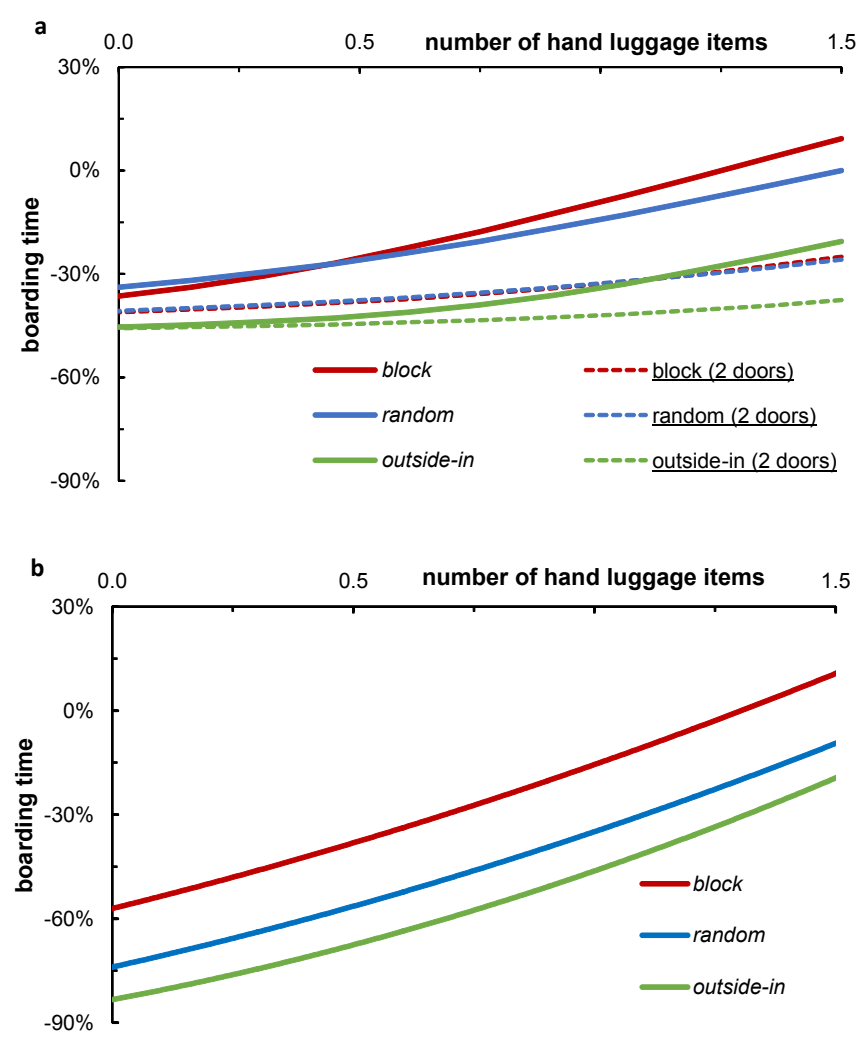

Figure 2. Boarding time reduction accompanied with a decreasing number of hand luggage items. (a) paxSim: the boarding time continuously decreases for both one-door and two-doors configuration; (b) PAXelerate confirm this trend but on a higher level.

\subsection{Distribution of Hand Luggage in the Cabin}

As the prior analysis clearly show, the reduction of hand luggage items is a good candidate to sustainably reduce the boarding time. Another approach aims the distribution of the hand luggage in the aircraft cabin (cf. $[25,56,57])$. In this paper, two strategies are evaluated: board high number of items first (bags first rule) and board passengers with a high number of hand luggage items in the rear of the aircraft (bags rear rule), despite any load/trim requirements. In Figure 3, the results of the evaluation are shown. Whereas PAXelerate indicates only a minor benefit/effort $( \pm 2 \%)$, paxSim demonstrates a significant benefit particularly for the bags-rear rule.

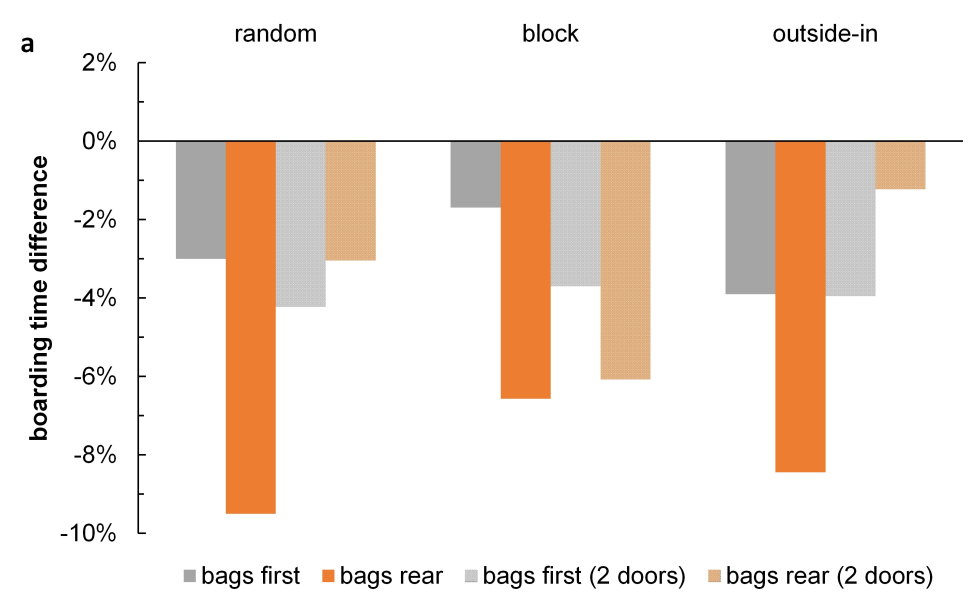

Figure 3. Cont. 


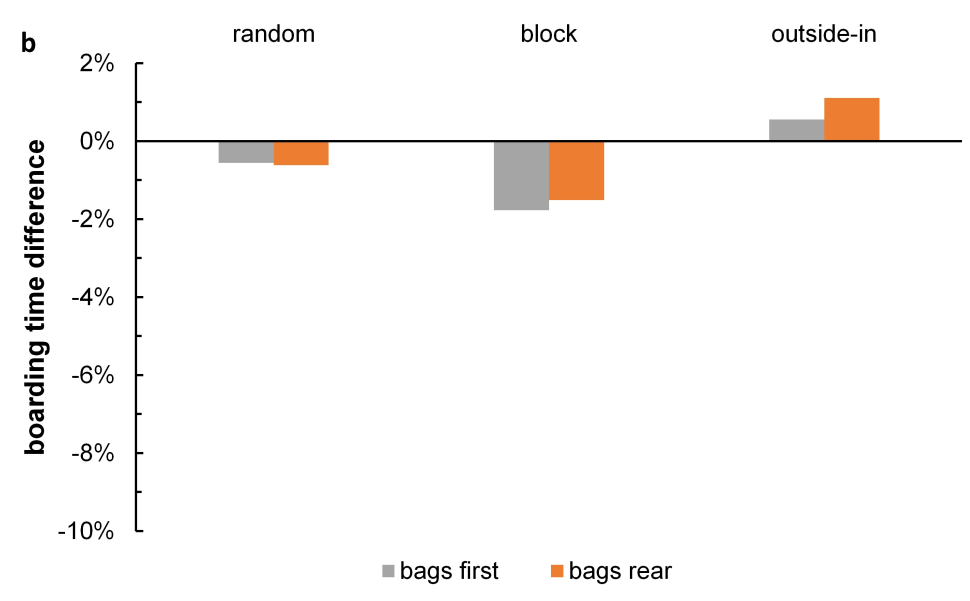

Figure 3. Boarding time differences applying bag-first and bags-rear rule on random, block, and outside-in boarding strategy for (a) paxSim and (b) PAXelerate.

\subsection{Infrastructural Changes}

Standard operational approaches to accelerating the boarding process mainly address the management of the passenger behavior by providing specific boarding sequences or the airline hand luggage regulation. A first infrastructural approach is to use the rear aircraft door, since this approach usually comes along with an apron position of the aircraft and a bus shuttle transfer (or walk boarding), which counteracts the idea of an optimized passenger sequencing. In fact, the use of the rear door results in substantially faster boarding times, even assume random boarding [21-23]. A significant change of the infrastructure has to overcome the negative impacts of a blocked aisle, caused by hand luggage storing or seat shuffling. With the innovative approach of foldable seats, the available infrastructure could be dynamically changed to support the boarding process by providing an extra space to allow two passengers to pass each other in a convenient way (details at $[30,45,58]$ ). Two variants of sideways foldable seat concepts exist: one model where the aisle seat is sliding over the middle seat, as proposed by Molon Labe Seating [46], and the other where the aisle seat sliding under the middle seat [59]. Figure 4 demonstrates the Side-Slip Seat (S3), where the aisle seat could be moved in the direction of the middle seat.

As simulation results and field trials show, a blocked aisle due to passengers storing baggage or entering their seat row impact the boarding most. The mitigate this negative impact, the available infrastructure could be dynamically changed to support the boarding process by providing a wider aisle using the innovative S3 technology. Thus, passengers will be able to pass each other in a convenient way. Due to the mandatory staggered seat design, the middle seat is 2 " wider than the aisle and window seats (aisle and window seats retain their standard width) and offers additional passenger comfort. Furthermore, a wider aisle enables a full-size wheelchair access down the aisle and airlines could address the needs of passengers with a reduced mobility [45]).

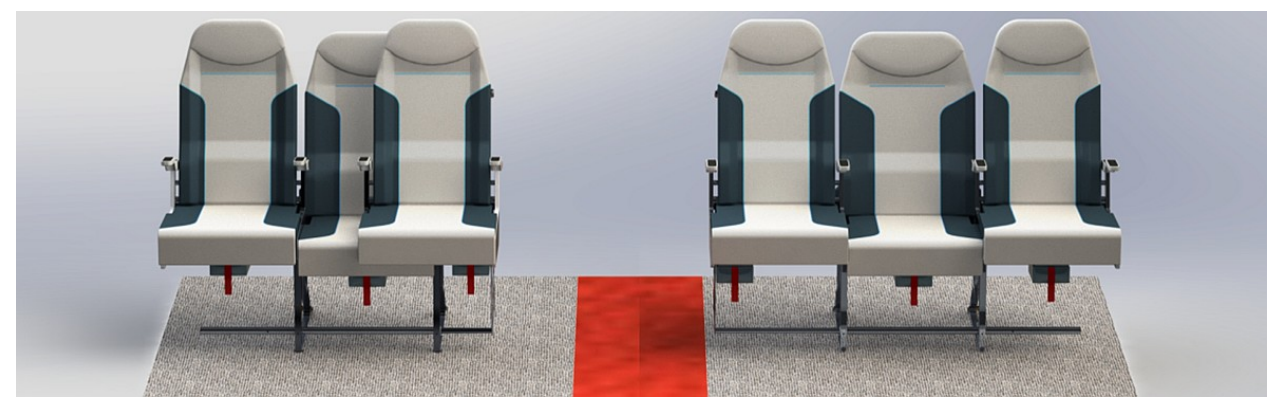

Figure 4. Innovative Side-Slip Seat seat in initial, folded condition (left) and unfolded operational condition (right), which is widen the aircraft aisle. 
The aisle seat will be in the initial position (folded) until one passenger wants to access the middle or aisle seat. The developed boarding models are adapted to allow the parallel movement of two passengers along the aisle. The dynamic status of the seat row (folded/unfolded) is implemented in both boarding models to enable/disable the parallel movement of passengers. If the seats on both sides of the row are in the initial condition, a second passenger can pass without reducing the walking speed. If only one side is unfolded, the speed is reduced by $50 \%$. If both sides are used by passengers, the standard boarding is active, where only one passenger is allowed to move in the aisle.

The concept of a cinema seat (Figure 5) was introduced by AIDA Development [55]. The aim of the seating concepts is to increase the moving space of passengers in the row and enhance their access to the overhead bins. If the aisle seat is not yet occupied, this seat configuration allows passengers to step into the seat row and to stow their hand luggage without blocking the aisle. In the case of seat row interferences when seated at the aisle, passengers can remain within their rows and stand up, which additionally reduce passengers interferences in the aisle.

Both seating concepts show improved boarding efficiency, as depicted in Figure 6, while slight advantages for the S3 seat are present. The stochastic paxSim demonstrate a conservative improvement of the boarding all boarding strategies (10-20\%), where PAXelerate demonstrate an optimistic evaluation of $30-60 \%$ faster boarding, if the new infrastructure is applied.

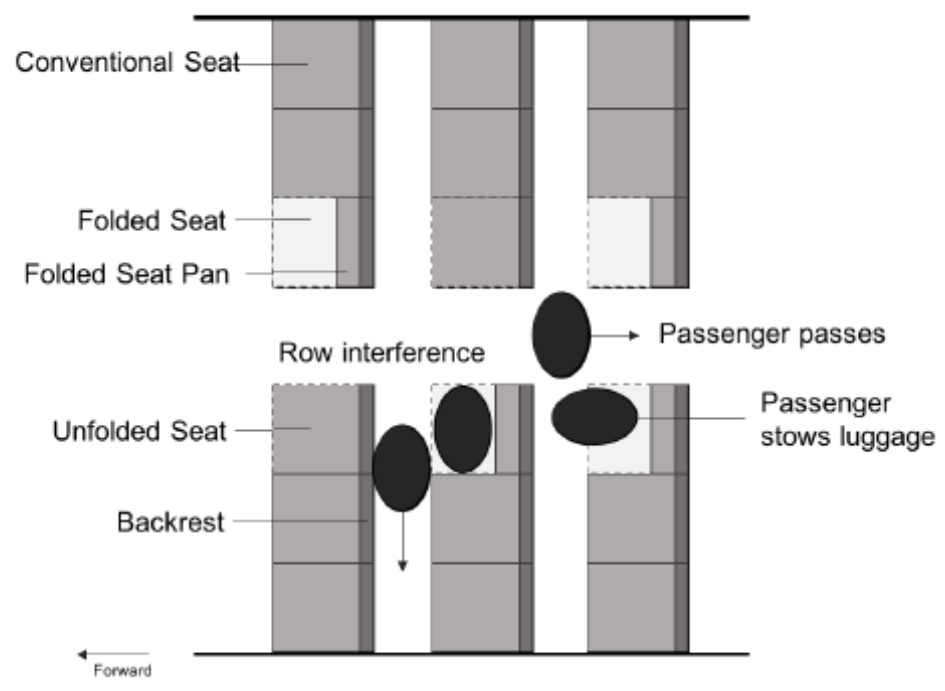

Figure 5. Concept of the cinema seat, which could be folded to provide additional space to store luggage in the overhead compartment and to efficiently enter the seat row (cf. [53]).

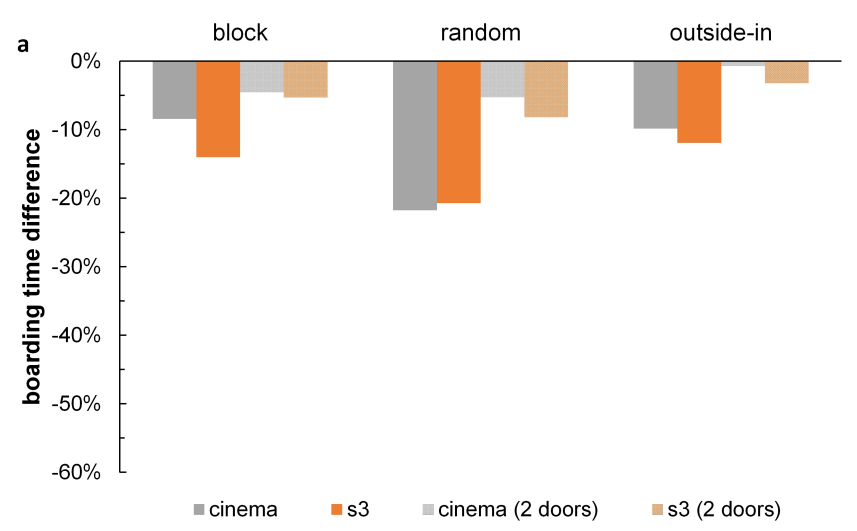

Figure 6. Cont. 


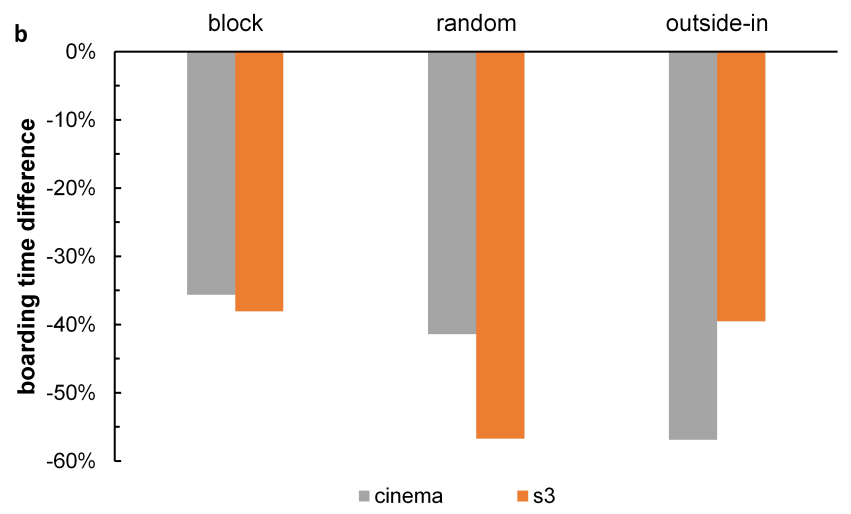

Figure 6. Comparison of boarding time impacts when the infrastructure in the cabin is changed by implementing the Side-Slip Seat (S3) and the cinema seat: (a) paxSim; (b) PAXelerate.

\subsection{Group with Two Members}

Passengers are traveling in groups and the groups size differs between business passengers and tourists. Business passengers tend to travel alone (73\% alone), whereas a tourist is often part of a group, only $19 \%$ of tourists are traveling alone [20]. A group of two passengers is implemented into paxSim to exemplarily model the effect of grouped passengers, assuming that group members are seated together. Group boarding will benefit from the fact that group members optimize their interactions themselves by choosing the most convenient sequence to enter the seat row: seats will be entered in the order of window, middle and aisle seat. This group behavior avoids unfavorable seat conditions, reduces time to take seats and mitigate the negative impact on the subsequently following passengers. In Figure 7, two exemplary group constellations are used in paxSim: a group with two members and a group with six members (sitting in two successive rows). While block and random strategy benefits from the increasing ratio of groups, the outside-in strategy are disturbed by group members.

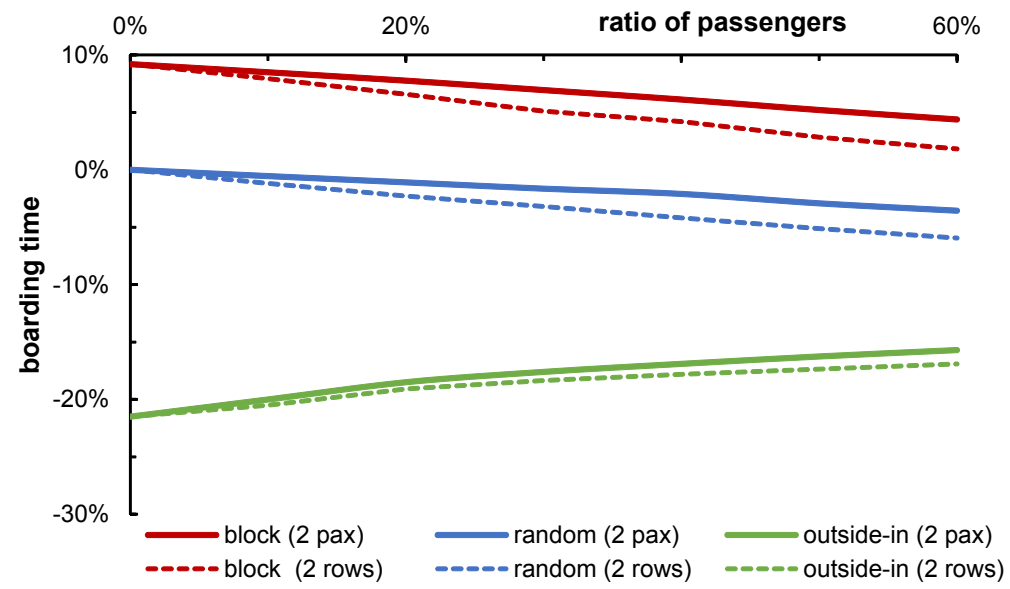

Figure 7. Impact to boarding time considering groups with two members and six members (two rows) using paxSim.

\section{Discussion of Results and Future Research}

There are many ideas to efficiently handle the future aircraft/passenger operations, to provide operational related design approaches, and to transfer the knowledge achieved with the A320 reference layout to twin-aisle or blended wing body configurations. The turnaround is the complex aircraft-airport-passenger interface and the key element for efficient operations. The airport facilities, such as check-in counters and boarding bridges, have to ensure a sufficient passenger flow to allow for 
a punctual completion of the boarding process. The predictability of the process time is challenging, due to the power of each individual passenger to influence the boarding process.

The foldable seats provide a backwards compatible solution for current aircraft. Certification and passenger acceptance (e.g., manageability, integration of inflight amenities and seating comfort) are the main aspects ensuring the operational applicability of the foldable seat concepts. Furthermore, the complex folding mechanism could result in higher maintenance efforts for the airline. Also the removal of the metal strap, which is located under standard seat and avoids hand luggage to move around the cabin, affects the stowage capability of the aircraft cabin. Considering today's high amount of hand luggage, this aspect is a major drawback of the foldable seat concept $[43,60]$. Furthermore, the mandatory seat belt demands a redesign to prevent getting blocked. We assumed in our evaluation that passengers are not blocking the aisle during unfolding their seats. However, this operational benefit could be significantly reduced if passengers will not follow this operational concept.

The new dynamic infrastructure approaches often comes with specific demands and appropriate changes in the procedural/operational design as well. In a first step, the boarding models have to be extended to cover the Side-Slip Seat and the cinema seat with their specific demand for a parallel movement in the aisle. In the prior evaluation only three boarding strategies are tested, but a deeper investigation into the S3 technology results in a new boarding strategy [45]. While current boarding strategies differentiate between the rear and the front of the aircraft, the new strategy differentiates between the left and the right side of the cabin. In Figure 8 this new left/right boarding strategy is shown: during half of the boarding progress, passengers are able to pass each other, which results in a $20 \%$ faster boarding (same level as outside-in boarding).

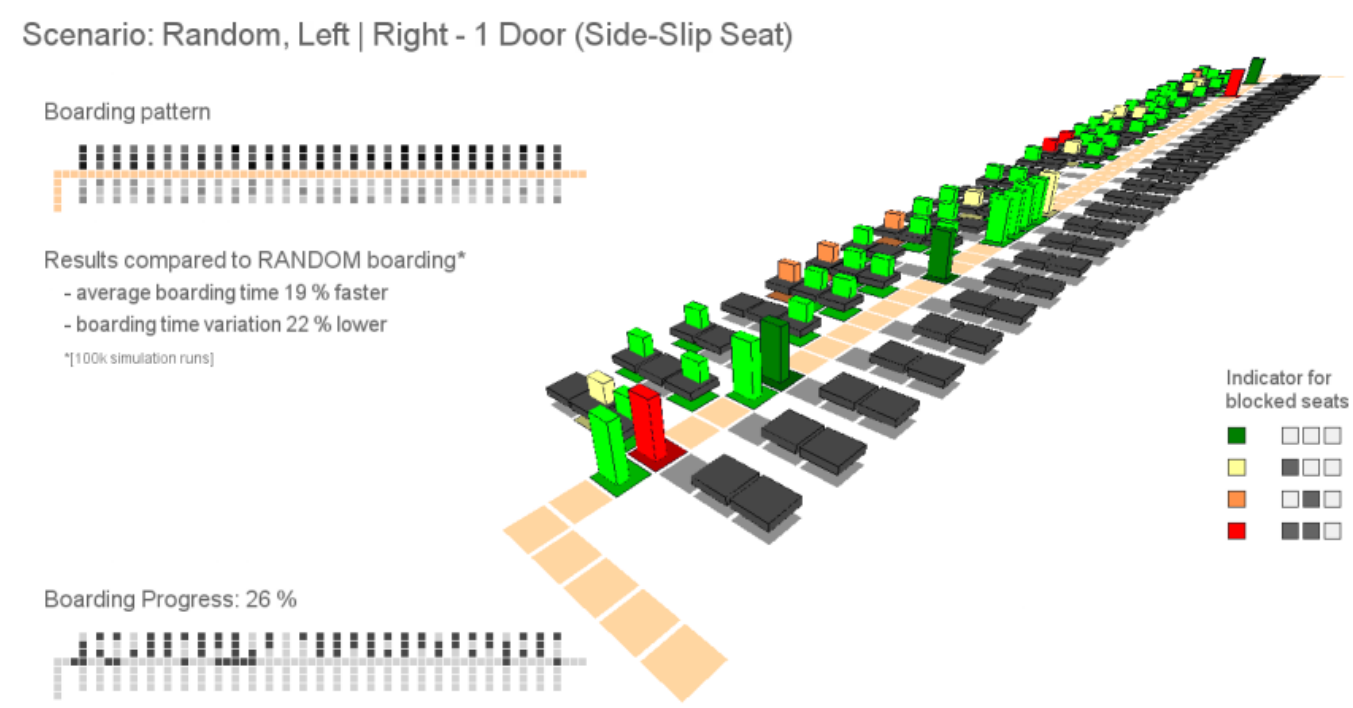

Figure 8. Implementation of Side-Slip Seat into the paxSim environment considering an appropriately adapted boarding strategy: one side of the aisle is boarded first.

The boarding process is not on the same level of criticality for most flights using a twin aisle aircraft, since delay can be compensated during a longer flight and scheduled ground time allowing transfer passenger to reach their connection. The twin-aisle cabin splits the passenger flow into two separate streams after the passengers have entered the aircraft. However, if one passenger queue reaches the door area due to aisle interferences (in the first rows) both aisles will be blocked. Lower boarding times can be accomplished when compared to single-aisle cabins accommodating an equal amount of passengers. An adaption of seat-based boarding schemes becomes necessary, especially in the case of an uneven number of middle seats and modified door positions.

The aircraft ground processes (turnaround) consists deboarding, catering, cleaning, fueling, boarding and the parallel processes of unloading and loading. In particular, the aircraft boarding is 
driven by the passengers' ability (experience) to follow the current boarding strategy (e.g., late arrivals, families, number hand luggage items, status passengers). For punctual airline operations, a reliable boarding time is essential to predict the end of the aircraft turnaround. In this context, the passenger-controlled boarding makes it difficult to reliably provide the final turnaround time, even if the boarding is already in progress [61,62].

It is expected, that in the future comprehensive cabin management systems will provide an enabling infrastructure to further improve the overall turnaround process. In a field trial with Eurowings at Cologne Bonn Airport, a new connected cabin concept was tested, using the aircraft seats (free or occupied) and the aisle floor (passenger position) as a sensor network. This sensor environment was successfully used for monitoring and evaluation of aircraft boarding progress in the operational environment. Figure 9 exhibits the test setup inside the Eurowings Airbus A319. The installed sensors in the cabin provide real-time status information about the aircraft boarding progress. These datasets are used as an input for a evaluation of current boarding complexity [61], which will be enable a reliable prediction of current boarding time in the future [62]. In combination with an integrated management of airport, airline and aircraft information, boarding could be a transparent progress in the future the with the ability to appropriately react to significant deviations from the planned progress.
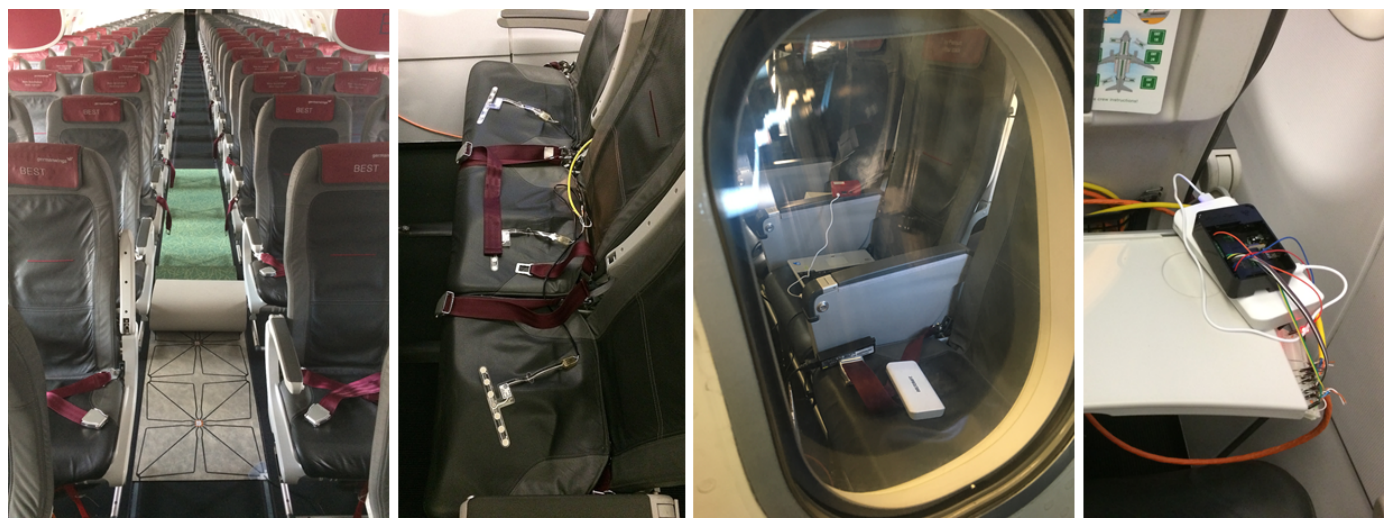

Figure 9. Sensor environment in a field trial environment: sensor floor, seat sensors, central processing unit, seat processing unit (from left to right).

Beside new technologies and procedures for the passenger boarding, the key element is still unsolved: the boarding is controlled by the passenger. Each passenger is able to significantly influence the whole aircraft boarding. Therefore the seatNow concept was developed by Schultz, which enables a dynamic seat allocation and brings the control back to the aircraft operator. Implementing seatNow, passengers get their seat assignments as recently as they scan their boarding passes (see Figure 10). The developed algorithm calculates the current most appropriate seat position in the aircraft, considering specific passenger preferences (e.g., status, group size, seat position) and the current progress of boarding (data provided by the sensor network and evaluated with a complexity metric [61]). During the field test with Eurowings seatNow was successfully tested against random and outside-in boarding strategies (definitions in [23]), taking also operational disturbances into account (e.g., groups). In this context, the random strategy provides a reference time for standard boarding and the outside-in strategy provides a reference time for fast boarding. As expected outside-in boarding was $13 \%$ faster than random boarding and seatNow was $22 \%$ faster than random boarding. 


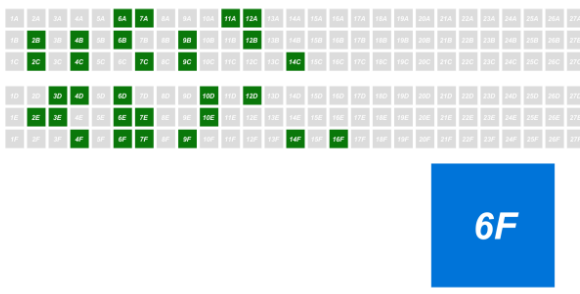

(a)

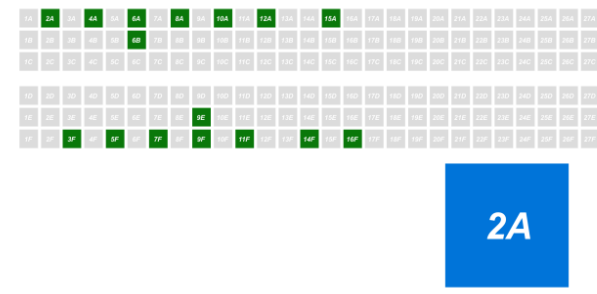

(b)

Figure 10. Dynamic seat allocation considering individual set preferences (e.g., passenger groups) and operational optimizations. (a) Random boarding sequence used as reference measurement. Currently assigned seat is 6F (row 6, window seat, left side); (b) Exemplarily optimized boarding strategy proposed by Steffen [24]. Currently assigned seat is $2 \mathrm{~A}$ (row 2, window seat, right side).

\section{Conclusions}

We provided a comparison of two model approaches, paxSim and PAXelerate, to reliably consider the individual behavior of passengers during aircraft boarding. The applications covered infrastructural changes and future technologies/procedures for aircraft boarding. The two model approaches showed similar overall trends but with deviations in the absolute benefit evaluation. A decreasing boarding time was identified, when the number of hand luggage items was reduced as well as when passengers with larger bags were seated in the rear cabin. The two innovative concepts of dynamic seats configuration (Side-Slip Seat and foldable cinema seat) resulted in a significantly faster boarding through providing extra space, which allowed passengers to pass each other in a convenient way. Furthermore, the paxSim implementation was used to quantify the impact of passenger groups. We exemplarily tested three prominent boarding strategies (random, block, and outside-in boarding) with a group of two passengers. While the random and block strategy benefited from the increasing ratio of groups, the outside-in strategy was disturbed by group members and showed a negative impact. Our contribution closed with a discussion of the achieved results and a brief outline about future connected aircraft cabin and boarding concepts providing a costumer-convenient dynamic seat allocation.

Author Contributions: All authors contributed equally to this work.

Funding: This research received no external funding.

Conflicts of Interest: The authors declare no conflict of interest.

\section{References}

1. Airbus. Global Market Forecast-Flying by Numbers; Airbus: Toulouse, France, 2017.

2. Boeing. Current Market Outlook 2017-2036; Boeing: Seattle, WA, USA, 2017.

3. European Commission. Flightpath 2050-Europe's Vision for Aviation; European Commission: Brussels, Belgium, 2011.

4. Montlaur, A.; Delgado, L. Flight and passenger delay assignment optimization strategies. Transp. Res. Part C Emerg. Technol. 2017, 81, 99-117. [CrossRef]

5. Oreschko, B.; Schultz, M.; Fricke, H. Skill Analysis of Ground Handling Staff and Delay Impacts for Turnaround Modeling. Air Transp. Oper. 2011, 310-318.

6. Oreschko, B.; Kunze, T.; Schultz, M.; Fricke, H.; Kumar, V.; Sherry, L. Turnaround Prediction with Stochastic Process Times and Airport specific Delay Pattern. In Proceedings of the 5th International Conference on Research in Airport Transportation, ICRAT, Berkeley, CA, USA, 22-26 January 2012.

7. Fricke, H.; Schultz, M. Delay Impacts onto Turnaround Performance. In Proceedings of the 8th USA/Europe Air Traffic Management Research and Development Seminar, Napa, CA, USA, 29 June-2 July 2009. 
8. Ivanov, N.; Netjasov, F.; Jovanović, R.; Starita, S.; Strauss, A. Air Traffic Flow Management slot allocation to minimize propagated delay and improve airport slot adherence. Transp. Res. Part A Policy Pract. 2017, 95, 183-197. [CrossRef]

9. Du, J.Y.; Brunner, J.O.; Kolisch, R. Planning towing processes at airports more efficiently. Transp. Res. Part E Logist. Transp. Rev. 2014, 70, 293-304. [CrossRef]

10. Rosenow, J.; Fricke, H.; Schultz, M. Air traffic simulation with 4D multi-criteria optimized trajectories. In Proceedings of the 2017 Winter Simulation Conference (WSC), Las Vegas, NV, USA, 3-6 December 2017; pp. 2589-2600.

11. Niklaß, M.; Lührs, B.; Grewe, V.; Dahlmann, K.; Luchkova, T.; Linke, F.; Gollnick, V. Potential to reduce the climate impact of aviation by climate restricted airspaces. Transp. Policy 2017. [CrossRef]

12. Rosenow, J.; Lindner, M.; Fricke, H. Impact of climate costs on airline network and trajectory optimization: A parametric study. CEAS Aeronaut. J. 2017, 8, 371-384. [CrossRef]

13. Rosenow, J.; Fricke, H.; Luchkova, T.; Schultz, M. Minimizing contrail formation by rerouting around dynamic ice-supersaturated regions. Aeronaut. Aerosp. Open Access J. 2018, 2, 105-111.

14. Rosenow, J.; Schultz, M. Coupling of Turnaround and Trajectory Optimization in an Air Traffic Simulation. In Proceedings of the 2018 Winter Simulation Conference (WSC), Gothenburg, Sweden, 9-12 December 2018.

15. Okwir, S.; Ulfvengren, P.; Angelis, J.; Ruiz, F.; Núñez Guerrero, Y.M. Managing turnaround performance through Collaborative Decision Making. J. Air Transp. Manag. 2017, 58, 183-196.

16. Mota, M.M.; Boosten, G.; De Bock, N.; Jimenez, E.; de Sousa, J.P. Simulation-based turnaround evaluation for Lelystad Airport. J. Air Transp. Manag. 2017, 64, 21-32. [CrossRef]

17. Fuchte, J. Enhancement of Aircraft Cabin Design Guidelines with Special Consideration of Aircraft Turnaround and Short Range Operations. Ph.D. Thesis, Technische Universität Hamburg-Harburg, Hamburg, Germany, 2014.

18. Marelli, S.; Mattocks, G.; Merry, R. The Role of Computer Simulation in Reducing Airplane Turn Time. Available online: https://www.boeing.com/commercial/aeromagazine/aero_01/textonly/t01txt.html (accessed on 24 October 2018).

19. Schultz, M. Entwicklung eines Individuenbasierten Modells zur Abbildung des Bewegungsverhaltens von Passagieren im Flughafenterminal. Ph.D. Thesis, Technische Universität Dresden, Dresden, Germany, 2010.

20. Schultz, M.; Fricke, H. Managing Passenger Handling at Airport Terminal. In Proceedings of the 9th USA/Europe Air Traffic Management Research and Development Seminar, Berlin, Germany, 14-17 June 2011.

21. Schultz, M.; Schulz, C.; Fricke, H. Efficiency of Aircraft Boarding Procedures. In Proceedings of the 3rd International Conference on Research in Airport Transportation (ICRAT), Fairfax, VA, USA, 1-4 June 2008; pp. 371-377.

22. Schultz, M.; Kunze, T.; Fricke, H. Boarding on the critical path of the turnaround. In Proceedings of the 10th USA/Europe Air Traffic Management Research and Development Seminar, Chicago, IL, USA, 10-13 June 2013.

23. Schultz, M. Implementation and application of a stochastic aircraft boarding model. Transp. Res. Part C Emerg. Technol. 2018, 90, 334-349. [CrossRef]

24. Steffen, J.H. Optimal boarding method for airline passengers. J. Air Transp. Manag. 2008, 14, 146-150. [CrossRef]

25. Milne, R.J.; Kelly, A.R. A new method for boarding passengers onto an airplane. J. Air Transp. Manag. 2014, 34, 93-100. [CrossRef]

26. Notomista, G.; Selvaggio, M.; Sbrizzi, F.; Di Maio, G.; Grazioso, S.; Botsch, M. A fast airplane boarding strategy using online seat assignment based on passenger classification. J. Air Transp. Manag. 2016, 53, 140-149.

27. Zeineddine, H. A dynamically optimized aircraft boarding strategy. J. Air Transp. Manag. 2017, 58, $144-151$. [CrossRef]

28. Jaehn, F.; Neumann, S. Airplane boarding. Eur. J. Oper. Res. 2015, 244, 339-359. [CrossRef]

29. Schultz, M. Fast Aircraft Turnaround Enabled by Reliable Passenger Boarding. Aerospace 2018, 5, 8. [CrossRef]

30. Schmidt, M. A review of aircraft turnaround operations and simulations. Prog. Aerosp. Sci. 2017, 92, 25-38. [CrossRef]

31. Van Landeghem, H.; Beuselinck, A. Reducing passenger boarding time in airplanes: A simulation based approach. Eur. J. Oper. Res. 2002, 142, 294-308. [CrossRef] 
32. Ferrari, P.; Nagel, K. Robustness of Efficient Passenger Boarding Strategies for Airplanes. Transp. Res. Rec. J. Transp. Res. Board 2005, 1915, 44-54. [CrossRef]

33. Bachmat, E.; Elkin, M. Bounds on the performance of back-to-front airplane boarding policies. Oper. Res. Lett. 2008, 36, 597-601. [CrossRef]

34. Bazargan, M. A linear programming approach for aircraft boarding strategy. Eur. J. Oper. Res. 2007, $183,394-411$.

35. Chung, C. Simulation Design Approach for the Selection of Alternative Commercial Passenger Aircraft Seating Configurations. J. Aviat. Technol. Eng. 2012, 2, 3. [CrossRef]

36. Bachmat, E.; Berend, D.; Sapir, L.; Skiena, S.; Stolyarov, N. Analysis of Airplane Boarding Times. Oper. Res. 2009, 57, 499-513. [CrossRef]

37. Wald, A.; Harmon, M.; Klabjan, D. Structured deplaning via simulation and optimization. J. Air Transp. Manag. 2014, 36, 101-109. [CrossRef]

38. Van den Briel, M.H.L.; Villalobos, J.R.; Hogg, G.L.; Lindemann, T.; Mulé, A.V. America West Airlines Develops Efficient Boarding Strategies. Interfaces 2005, 35, 191-201. [CrossRef]

39. Bachmat, E.; Khachaturov, V.; Kuperman, R. Optimal back-to-front airplane boarding. Phys. Rev. E Stat. Nonlinear Soft Matter Phys. 2013, 87, 062805. [CrossRef] [PubMed]

40. Soolaki, M.; Mahdavi, I.; Mahdavi-Amiri, N.; Hassanzadeh, R.; Aghajani, A. A new linear programming approach and genetic algorithm for solving airline boarding problem. Appl. Math. Model. 2012, 36, 4060-4072. [CrossRef]

41. Frette, V.; Hemmer, P.C. Time needed to board an airplane: A power law and the structure behind it. Phys. Rev. E Stat. Nonlinear Soft Matter Phys. 2012, 85, 011130. [CrossRef] [PubMed]

42. Tang, T.Q.; Wu, Y.H.; Huang, H.J.; Caccetta, L. An aircraft boarding model accounting for passengers' individual properties. Transp. Res. Part C Emerg. Technol. 2012, 22, 1-16. [CrossRef]

43. Schmidt, M.; Heinemann, P.; Hornung, M. Boarding and Turnaround Process Assessment of Single- and Twin-Aisle Aircraft. In Proceedings of the 55th AIAA Aerospace Sciences Meeting, Grapevine, TX, USA, 9-13 January 2017.

44. Schmidt, M.; Heinemann, P. Improving the Boarding Performance of Regional Aircraft. In Proceedings of the 17th AIAA Aviation Technology, Integration, and Operations Conference, Denver, CO, USA, 5-9 June 2017.

45. Schultz, M. Dynamic change of aircraft seat condition for fast boarding. Transp. Res. Part C Emerg. Technol. 2017, 85, 131-147. [CrossRef]

46. Molon Labe Seating. Side-Slip Seat. Available online: https://www.airlineseats.biz/ (accessed on 26 September 2018).

47. Steffen, J.H.; Hotchkiss, J. Experimental test of airplane boarding methods. J. Air Transp. Manag. 2012, 18 , 64-67.

48. Kierzkowski, A.; Kisiel, T. The Human Factor in the Passenger Boarding Process at the Airport. Procedia Eng. 2017, 187, 348-355. [CrossRef]

49. Miura, A.; Nishinari, K. A passenger distribution analysis model for the perceived time of airplane boarding/deboarding, utilizing an ex-Gaussian distribution. J. Air Transp. Manag. 2017, 59, 44-49. [CrossRef]

50. Schultz, M. Field Trial Measurements to Validate a Stochastic Aircraft Boarding Model. Aerospace 2018, 5, 27. [CrossRef]

51. Gwynne, S.M.V.; Senarath Yapa, U.; Codrington, L.; Thomas, J.R.; Jennings, S.; Thompson, A.J.L.; Grewal, A. Small-scale trials on passenger microbehaviours during aircraft boarding and deplaning procedures. J. Air Transp. Manag. 2018, 67, 115-133. [CrossRef]

52. Schultz, M. Stochastic Transition Model for Pedestrian Dynamics. In Pedestrian and Evacuation Dynamics 2012; Springer International Publishing: Cham, Switzerland, 2013; pp. 971-985.

53. Schmidt, M.; Engelmann, M.; Brügge-Zobel, T.; Hornung, M.; Glas, M. PAXelerate-An Open Source Passenger Flow Simulation Framework for Advanced Aircraft Cabin Layouts. In Proceedings of the 54th AIAA Aerospace Sciences Meeting, San Diego, CA, USA, 4-8 January 2016.

54. Schmidt, M. PAXelerate Release 1.0, Github Repository. Available online: http://www.paxelerate.com (accessed on 26 September 2018).

55. Sii Engineering. Foldable Passenger Seat I SII Group-The Aida Way. Available online: http://www.siiengineering.de (accessed on 26 September 2018).

56. Qiang, S.J.; Jia, B.; Xie, D.F.; Gao, Z.Y. Reducing airplane boarding time by accounting for passengers' individual properties: A simulation based on cellular automaton. J. Air Transp. Manag. 2014, 40, 42-47. [CrossRef] 
57. Milne, R.J.; Salari, M. Optimization of assigning passengers to seats on airplanes based on their carry-on luggage. J. Air Transp. Manag. 2016, 54, 104-110. [CrossRef]

58. Schultz, M. The Seat Interference Potential as an Indicator for the Aircraft Boarding Progress. SAE Tech. Pap. 2017. [CrossRef]

59. Isikveren, A.T.; Seitz, A.; Vratny, P.C.; Pornet, C.; Plötner, K.O.; Hornung, M. Conceptual studies of universally-electric systems architectures suitable for transport aircraft. In Deutscher Luft-und Raumfahrt Kongress; DLRK: Berlin, Germany, 2012.

60. Schmidt, M.; Engelmann, M.; Rothfeld, R.; Hornung, M. Boarding Process Assessment of Novel Aircraft Cabin Concepts. In Proceedings of the 30th International Congress of the Aeronautical Sciences, Daejoun, Korea, 25-30 September 2016.

61. Schultz, M. A metric for the real-time evaluation of the aircraft boarding progress. Transp. Res. Part C Emerg. Technol. 2018, 86, 467-487. [CrossRef]

62. Schultz, M.; Reitmann, S. Machine learning approach to predict aircraft boarding. Transp. Res. Part C Emerg. Technol. 2018, in press.

(C) 2018 by the authors. Licensee MDPI, Basel, Switzerland. This article is an open access article distributed under the terms and conditions of the Creative Commons Attribution (CC BY) license (http://creativecommons.org/licenses/by/4.0/). 